\title{
Análise eletromiográfica dos membros inferiores em cinco posições de ciclismo indoor*
}

\section{Electromyographic analysis of lower limbs in five indoor cycling positions}

Márcio Rabelo Mota ${ }^{1}$ Sandro Nobre Chaves ${ }^{2}$

Filipe Dinato de Lima $^{3}$ Adriano Loboissiere Botelho ${ }^{4}$ Ricardo Jacó de Oliveira ${ }^{5}$
Recebido em: 25/04/2016.

Aprovado em: 04/11/2016.

1 Professor do Centro Universitário de Brasília - UniCEUB - Brasília DF/Brasil. E-mail: marciormota@gmail.com

2 Professor da Universidade de Brasília.

Professor da Universidade de Brasília.

4 Estudante de Educação Física do Centro Universitário de Brasília.

5 Professor da Universidade de Brasília.

\section{Resumo}

Com a evolução das bicicletas, surgiu a bicicleta estacionária, ou chamada cicloergômetros, que é voltada para uso em salas de avaliação física. O objetivo deste artigo foi verificar a ativação dos músculos vasto lateral (VL), vasto medial (VM) e reto femoral (RF) em cinco diferentes posições de ciclismo indoor, por meio da eletromiografia (EMG). A amostra foi composta por 10 indivíduos do sexo masculino. As atividades elétricas dos músculos foram coletadas durante o movimento de pedalada em bicicleta específica de ciclismo estacionário, tendo os participantes se posicionado em cinco posições diferentes, para avaliação do VL, VM e RF. De acordo com as posições corporais, foram observadas diferenças nas respostas EMG para cada um dos músculos estudados. Em relação à análise das respostas EMG dos membros inferiores em cinco diferentes posições em ciclistas indoor, foram encontradas diferenças significativas no músculo RF.

Palavras-chave: Ciclo ergômetro. Eletromiografia. Ativação muscular. Exercício físico.

\begin{abstract}
Evolution of bicycles, one stationary bike came, or Call cycle ergometers, which are aimed paragraph OSU evaluation rooms física. O objective of this study was to verify the activation of the vastus lateralis muscles (VL), vastus (VM) femoral and rectum ( RF) at five different positions within cycling through the Electromyography (EMG). The sample consisted of 10 male individuals. As Electrical Activities of Muscles Were collected during the pedaling motion in Specific bike stationary cycling, OS Participants tendon positioned in Five Different Positions, paragraph rating to VL, VM and RF. From According to how Body Positions Were observed differences in responses EMG paragraph Each hum of the studied muscles. The analysis of the lower limbs EMG Responses in five different positions in indoor cyclists Were found significant differences not RF muscle.
\end{abstract}

Keywords: Syphilis. Ergometer cycle. Electromyography. Muscle activation. Exercise. 


\section{Introdução}

Com a evolução das bicicletas, surgiu a bicicleta estacionária, ou chamada cicloergômetros, que são voltadas para uso em salas de avaliação física, residências, academias de ginásticas e salas de aulas coletivas (RIENDA et al., 2012; SILVA; OLIVEIRA, 2002).

O cicloergômetro foi criado para quantificar a capacidade do trabalho muscular realizado pelas pessoas por meio de testes laboratoriais e vem sendo utilizado como ferramenta para determinar os parâmetros fisiológicos e biomecânicos em ciclistas. Por ser o ciclismo uma atividade física que demanda a utilização de grandes grupos musculares associados a um reduzido grau de impacto sobre as articulações, tornou-se um dos exercícios mais utilizados para a avaliação clínica de pacientes, bem como, para o desenvolvimento de aptidão cardiorrespiratória nos indivíduos saudáveis (DIAS et al., 2007).

A eficiência muscular de um indivíduo durante a pedalada pode ser modificada por fatores materiais, tais como a altura do banco, o tamanho do pedivela, o posicionamento na bicicleta ou mesmo o uso de sapatilhas de ciclismo (MESQUITA FILHO; CASTRO, 2011).

Independentemente da finalidade a qual se destina, lazer ou competição, a bicicleta deve ser ajustada de acordo com as medidas corretas para que sejam alcançados os objetivos pretendidos. Tais regulagens devem se ater não somente com relação ao quadro da bicicleta, considerada a principal medida ao se escolher uma bicicleta, mas também com relação ao posicionamento do selim em relação às posições verticais e horizontais, dentre outras partes ajustáveis (KLEINPAUL et al., 2010).

Por exemplo, o posicionamento do selim muito baixo e/ou avançado pode gerar uma flexão ou "movimentação" médio-lateral excessiva do joelho e, por sua vez, o posicionamento do selim muito alto e/ou recuado pode ocasionar uma tensão excessiva dos músculos posteriores da coxa, que são capazes de acarretar lesões tais como tendinite (PINZON et al., 2010).

Sabe-se que o posicionamento errado do selim gera maior consumo de oxigênio, assim como um maior trabalho muscular, ocasionando menor eficiência. Certos autores indicam que o ângulo do joelho, durante o movimento de flexão, não pode ser maior do que $65^{\circ}$ (sessenta e cinco graus) e, no movimento de extensão, é indicado que o joelho permaneça próximo a $150^{\circ}$ (cento e cinquen- (treze graus). Por sua vez, a ocorrência da extensão total do joelho durante a pedalada dificulta o desempenho dos músculos flexores do joelho (ALVES, 2010).

No que tange ao movimento dos joelhos durante a pedalada, observa-se que o movimento de extensão é o mais impactante, já que a flexão do joelho é realizada de forma passiva por não ter maiores participações dos seus flexores (SILVA et al., 2012).

A oscilação das pedaladas são compostas fundamentalmente por duas fases; a fase ascendente, na qual os pedais da bicicleta se deslocam de baixo para cima, e a fase descendente, onde há deslocamento do pedal de cima para baixo, fase que incide maior potência (SILVA et al., 2012).

Destaca-se, ainda, que, na fase descendente da pedalada, ativam-se os músculos glúteo máximo (GM), glúteo médio $(\mathrm{GL})$, vasto intermédio $(\mathrm{VI})$, vasto lateral (VL), vasto medial (VM), reto femoral (RF), gastrocnêmio (MG) e sóleo (SOL), ocorrendo uma extensão do quadril e joelho, enquanto, na fase ascendente, são ativados os músculos ilipsoas, isquiotibiais e tibial anterior, ocorrendo uma flexão do quadril e joelho (FERMINO, 2008).

A atividade muscular pode ser mensurada por meio da Eletromiografia de superfície (EMG), que é o método utilizado para estudar a atividade neuromuscular, em que o sinal elétrico é amplificado e registrado por meio de gráfico, permitindo avaliar o grau e duração da atividade muscular, ocorrência de fadiga, alteração da composição das unidades motoras, dentre outros fatores. A Eletromiografia (EMG) tem sido utilizada, largamente, com a finalidade de estudar a atividade muscular e a coordenação neuromuscular no ciclismo (CRUZ, 1998; FERREIRA et al., 2010; DIEFENTHAELER; VAZ, 2008).

Estudos apontam acréscimo na ativação muscular em função da diminuição da altura do selim, mais especificamente, com relação aos músculos isquiotibiais e quadríceps, mas de uma forma geral, nos estudos realizados com EMG em alturas distintas do selim, os resultados se apresentam de forma variada. Por exemplo, os estudos de Ericson et al. (1985), apontam que o aumento na altura do selim gera uma maior ativação dos músculos GM e SOL, entretanto, no estudo de Houtz e Fischer (1959), tal situação revelou menor ativação no mesmo grupo de músculos (DIEFENTHAELER et al., 2008).

Assim, qualquer alteração na posição do ciclis- 
ta afetará, diretamente, o seu desempenho, sendo que, qualquer ajuste, ainda que pequeno, pode influenciar de forma distinta cada ciclista, razão pela qual não se pode generalizar os efeitos dessas alterações, devendo ser investigada cada uma delas, de forma individualizada (DIEFENTHAELER et al., 2008).

Portanto, o objetivo deste estudo é verificar a ativação dos músculos inferiores Vasto Lateral, Vasto Medial e Reto Femoral em cinco diferentes posições de ciclismo indoor, por meio da Eletromiografia.

\section{Materiais e Métodos}

\subsection{Tipo de Estudo}

O delineamento do estudo é caracterizado por uma pesquisa transversal do tipo comparativo-casual.

\subsection{Amostra}

A amostra foi composta por 10 indivíduos do gênero masculino, com idade média de $24,6( \pm 3,4)$ anos de idade. Quanto às medidas antropométricas, esta amostra apresentou massa corporal média de 75,50 $( \pm 11,76) \mathrm{Kg}$ e estatura média de $175( \pm 8)$ centímetros.

O grupo foi escolhido pela facilidade de controle de aderência ao processo (teste e avaliações) e pelo controle dos mecanismos de intervenção (praticantes de ciclismo estacionário).

O projeto foi submetido ao Comitê de Ética e Pesquisa (CEP-UniCEUB), obtendo aprovação com o número do parecer 118.627 em 05/10/2012.

\subsubsection{Critérios de Inclusão}

Foram incluídos participantes do gênero masculino, praticantes de ciclismo estacionário há pelo menos 2 (dois) meses, com vivência das posições e técnicas utilizadas nas aulas e que concordaram em assinar o Termo de Consentimento Livre e Esclarecido (TCLE).

\subsubsection{Critérios de Exclusão}

Foram excluídos aqueles que estavam utilizando medicamentos e/ou recursos ergogênicos nutricionais, que possuíam problemas de saúde ou limitações osteomioarticulares ou que não seguiram as recomendações para a realização dos testes.

\subsection{Local e Instrumento das Coletas de Dados}

Todas as coletas foram realizadas no laboratório de fisiologia do UniCEUB. A coleta de dados foi realizada em 1 (um) dia, no período matutino, anteriormente à realização de qualquer atividade física, para que não houvesse interferência da rotina diária na amostra.

O movimento da pedalada em diferentes posições de uma aula de ciclismo estacionário foi realizado em uma bicicleta específica de ciclismo estacionário, da marca Probike.

Para a aquisição dos dados foi utilizado um aparelho eletromiográfico (EMG) de 8 canais EMG System do Brasil, EMG_800_USB, com filtragem butterwoth " $\mathrm{f}$ " inferior 10 , " $\mathrm{f}$ " superior 500 , ordem 4 , sinais entre $-2000 \mathrm{~Hz}$ a $2000 \mathrm{~Hz}$, com frequência de amostragem de 30 segundos por quadro. Foram, ainda, utilizados os eletrodos Meditrace 200 de ECG de superfície passivos e autoadesivos com $2 \mathrm{~cm}$ cada, que foram colocados em configuração bipolar, fixados nos músculos que constituem a região do quadríceps e o eletrodo de referência foi fixado na vértebra C7, de acordo com as diretrizes do projeto SENIAM (SURFACE ELECROMYOGRAPHY FOR THE NON-INVASIVE ASSESSMENT OF MUSCLES, 2012).

A análise dos dados foi realizada utilizando um computador da marca LG com um processador Intel Core Duo E6750 2,66 GHz, 2GB RAM e com 148 GB HD.

Todos os equipamentos estavam devidamente calibrados.

\subsection{Procedimentos do estudo}

Para realização do estudo, foi utilizado o seguinte protocolo:

\subsubsection{Posicionamento dos Eletrodos}

Os eletrodos foram posicionados nos músculos VL, VM e RF. Para o músculo VL, os eletrodos foram colocados na distância de 2/3 na linha da suprailíaca superior até o lado lateral da patela. Para o VM, os eletrodos foram colocados na distância de $80 \%$ da linha entre a suprailíaca anterior superior e do espaço articular na frente da borda anterior do ligamento colateral medial. Para o músculo RF, os eletrodos foram colocados na distância de $50 \%$ da linha longitudinal a partir da suprailíaca superior até a parte superior da patela (SURFACE ELECROMYOGRAPHY FOR THE NON-INVASIVE ASSESSMENT OF MUSCLES, 2012).

\subsubsection{Ajuste da bicicleta adaptado de Carvalho (2005)}

-Altura do banco (selim) - o banco foi posicionado na altura do osso do quadril (crista ilíaca). O participante subiu na bicicleta e pedalou lentamente com as mãos apoiadas no guidom. $\mathrm{O}$ joelho ficou levemente flexionado quando o pé passava próximo ao solo. Esse 
ângulo girou em torno de $25^{\circ}$. O joelho não ficou muito flexionado e nem completamente estendido.

-Distância do banco (selim) - o participante posicionou-se sentado na bicicleta com os pés paralelos (pés de vela paralelos ao solo), traçando-se uma linha imaginária na face anterior da patela com o eixo central do pedal.

-Altura do guidom - o guidom ficou na mesma altura do banco (selim).

\subsubsection{Coleta de dados}

As atividades elétricas dos músculos foram coletadas durante o movimento de pedalada em bicicleta específica de ciclismo estacionário para a realização do movimento. A cadência das pedaladas foi fixada em 80 rotações por minuto em carga moderada, de acordo com a percepção subjetiva do esforço da escala de Borg. Os movimentos foram executados durante 30 segundos. No entanto, foram coletados apenas os últimos 20 segundos de cada posição, havendo intervalo de 10 segundos entre as coletas de cada uma das posições (CANDOTTI et al., 2012).

A coleta foi realizada em cinco momentos distintos:

1.posição: os participantes pedalaram sentados com as mãos na lateral do guidom, na $2^{\mathrm{a}}$ posição, e com altura do banco permitindo um grau de flexão do joelho de $25^{\circ}$ graus conforme literatura;

2.Segunda posição: os participantes pedalaram em pé com as mãos ao lado do guidom, na $2^{\mathrm{a}}$ posição;

3.posição: os participantes pedalaram em pé com as mãos nas extremidades do guidom;

4.Quarta posição: os participantes pedalaram novamente sentados com a mão ao lado do guidom, na $2^{\text {a }}$ posição, alterando-se a posição do banco, em 2 (dois) furos abaixo da posição do banco inicial;
5. Quinta posição: os participantes pedalaram sentados com as mãos ao lado do guidom, na $2^{\text {a }}$ posição, com o banco posicionado em 1 (um) furo mais alto que a posição inicial.

6.A atividade EMG foi expressa pela média do sinal retificado (RMS).

\subsection{Análise estatística}

Foi realizada análise exploratória dos dados e utilizada a estatística descritiva, média \pm desvio-padrão, para organizar e apresentar os dados. A caracterização amostral foi realizada por meio de cálculo da média e desvio padrão das variáveis: idade, estatura e peso. Para testar as variáveis dependentes (Eletromiografia dos músculos Vasto Lateral, Vasto Medial e Reto Femoral) nas diferentes posições corporais, foi realizada análise de variância para medidas repetidas (repeated measures ANOVA ONE-WAY). A significância estatística adotada para as análises foi de 95\% ( $\mathrm{p}<0,005)$. Ocorrendo diferença significativa em alguma das variáveis, testes de comparações múltiplas Bonferroni foram adotados para identificação de contrastes relevantes entre as médias. O software SPSS versão 17.0 foi utilizado para realização de todas as análises.

\section{Resultados}

Ao analisar as características referentes à eletromiografia (RMS em $\mathrm{mV}$ ) de acordo com a posição corporal, ou seja, nas posições sentado, em pé, em pé inclinado, sentado com o banco mais alto e sentado com o banco mais baixo, foram observadas diferenças significativas entre os momentos, conforme se observa na Quadro 1.

\begin{tabular}{|c|c|c|c|c|}
\hline \multirow{6}{*}{ RMS (mV) } & & Vasto Lateral & Vasto Medial & Reto Femoral \\
\hline & Sentado & $175,79 \pm 56,18$ & $130,98 \pm 53,38^{*}$ & $59,61 \pm 16,45$ \\
\hline & Pé & $202,72 \pm 43,35$ & $183,05 \pm 78,67$ & $134,46 \pm 48,62^{*}$ \\
\hline & Pé Inclinado & $228,25 \pm 53,80$ & $187,32 \pm 61,49$ & $150,93 \pm 92,00^{*}$ \\
\hline & Banco Baixo & $192,89 \pm 48,78$ & $171,84 \pm 38,17$ & $88,96 \pm 40,91$ \\
\hline & Banco Alto & $192,21 \pm 29,74$ & $161,48 \pm 34,30$ & $76,60 \pm 30,17$ \\
\hline \multicolumn{2}{|c|}{ Anova* } & $p=0,113$ & $\mathrm{p}=0,019$ & $p=0,002$ \\
\hline \multicolumn{5}{|c|}{$\begin{array}{l}\text { RMS = raiz quadrada média; } \\
\text { * Nível de significância do teste Anova para medidas repetidas }(p<0,05)\end{array}$} \\
\hline
\end{tabular}


Foi observado que, durante a pedalada, o músculo vasto lateral obteve maior incidência de ativação muscular para todas as posições examinadas, com $\mathrm{p}=0,113$, razão pela qual, não apresentou diferenças significativas.

O músculo que recebeu menor ativação muscular durante o movimento da pedalada foi o reto femoral para todas as posições estudadas, com $\mathrm{p}=0,002$.

$\mathrm{O}$ músculo vasto medial restou com incidência de ativação muscular mediana entre os demais músculos avaliados, com $\mathrm{p}=0,019$.

Os maiores índices obtidos no estudo foram apresentados quando os participantes se encontravam na posição em pé inclinado, o que implica na afirmativa de que nesta posição, o participante obteve maior ativação muscular em todos os músculos pesquisados.

Por outro lado, os menores resultados obtidos na pesquisa com relação aos músculos examinados revelaram-se na posição sentado, quando os participantes obtiveram os menores índices de ativação muscular.

Assim, os participantes realizaram maior esforço muscular, dentre os músculos estudados, quando estavam na posição em pé inclinado, e menor esforço, quando estavam na posição sentado, sendo necessário salientar que, quando a posição do selim esteve ajustada em posição mais alta ou mais baixo que a posição inicialmente adotada, houve aumento do índice RMS, conforme revela o quadro 1.

Extrai-se das informações obtidas que, embora não tenha havido maiores alterações nos valores encontrados nas ativações musculares para as posições estudadas no músculo vasto lateral e vasto medial, houve diferenças nos índices RMS colhidos para o músculo reto femoral de acordo com a variação das posições (Quadro 1).

Como esperado antes da coleta de dados, a alteração das posições aumentou a exigência da musculatura estudada.

\section{Discussão}

O objetivo deste estudo foi verificar as respostas das atividades eletromiográficas dos músculos do VL, $\mathrm{VM}$ e RF em diferentes posições e diferentes alturas do selim no ciclismo estacionário.

Os resultados demonstraram que a atividade muscular sofreu diferenças significativas no músculo reto femoral nas diferentes posições encontradas, sendo a posi- ção em pé, estatisticamente diferente da posição sentado $(\mathrm{p}=0,010)$ e de banco alto $(\mathrm{p}=0,017)$.

$\mathrm{O}$ músculo $\mathrm{VL}$, conforme relatado não obteve diferença significativa entre as posições examinadas $(\mathrm{p}=0,113)$, resultado que se assemelha ao resultado obtido por Alves (2010), que concluiu que em diferentes posições do selim o músculo VL obteve $\mathrm{p}>0,05$. Tais resultados, também, foram alcançados por Okano et al. (2005), que, em testes de Wingate, não encontraram diferenças significativas entre os valores RMS, tendo para o músculo VL obtido o resultado $\mathrm{p}=0,262$.

Em estudo análogo, Candotti et al (2004), ao avaliarem a ativação muscular do músculo VL, em teste de fadiga, verificaram que não foram encontradas diferenças significativas entre os resultados com relação aos valores RMS ( $\mathrm{p}=0,698)$.

O músculo VM restou com incidência de ativação muscular mediana entre os demais músculos avaliados, com $\mathrm{p}=0,019$ não sendo obtida resposta significativa. Resultado semelhante foi obtido por Alves (2010), que encontrou $\mathrm{p}>0,05$, assim como na pesquisa realizada por Okano et al. (2005) que encontraram p=0,282.

$\mathrm{O}$ músculo que recebeu menor ativação muscular durante o movimento da pedalada no presente estudo foi o RF para todas as posições avaliadas, com $p=0,002$, sendo o único músculo, dentre os estudados, que obteve resultado significativo. Resultados semelhantes foram encontrados por Dorel et al. (2009), que em estudo que avaliou a produção de força de atletas em diferentes posicionamentos, revelou diminuição da atividade do reto femoral (RF) em posição aerodinâmica $(\mathrm{p}<0,01)$, concluindo que referidas mudanças estriam relacionadas com a direção da aplicação da força na posição aerodinâmica.

No estudo já citado, realizado por Okano et al. (2005), ao compararem a ativação muscular no músculo RF, encontraram o $\mathrm{p}=0,351$ em testes de Wingate. Não obstante os resultados obtidos para o primeiro e segundo testes que não revelaram diferença significativa para o músculo RF, foram encontradas diferenças significantes entre os valores de RMS obtidos no terceiro e último teste, no qual o músculo $R F$ obteve $\mathrm{p}<0,05$.

Alves (2010), em seu estudo, obteve como resultado de suas coletas, situação adversa da evidenciada pela presente pesquisa com relação ao músculo RF, tendo encontrado $p>0,05$ e verificado que o músculo apresentou maior diferença quanto a sua ativação.

Quanto à análise neuromuscular dos músculos 
estudados, impende salientar que possuem particularidades entre si, quanto à composição de fibras, o RF apresenta maior proporção de fibras do tipo II em relação aos músculos VL e VM; e, quanto ao formato muscular, o VL e VM são músculos peniformes e o RF é fusiforme. A diferença anatômica da musculatura estudada pode influenciar e justificar os diferentes resultados obtidos com cada um dos músculos estudados (OKANO et al., 2005).

\section{Conclusão}

Conclui-se que, em relação à análise das respostas EMG dos membros inferiores em cinco diferentes posições em ciclistas indoor, foram encontradas diferenças significativas no músculo reto femoral (RF) na posição em pé em relação às posições sentado e banco alto. Além disso, os achados demonstraram que, na posição em pé inclinada, houve maior ativação de todos os músculos, porém, na posição sentada, todos os músculos apresentaram menor ativação.

Assim, perante os resultados obtidos, é possível afirmar que as alterações na altura do selim, bem como as diferentes posições estudadas, influenciam, diretamente, a ativação muscular, que, por sua vez, está ligada, diretamente, ao desempenho do atleta.

\section{Referências}

ALVES, E. G. Análise das respostas EMG lombar e de membros inferiores em dois diferentes ajustes no ângulo do selim em ciclistas de rua durante a pedalada. 2010. 67 f. Projeto de trabalho de conclusão de curso (Pós-graduação) - Universidade do Extremo Sul Catarinense, Criciúma, 2010.

CANDOTTI, C. T. et al. Atividade elétrica e força muscular dos extensores cervicais durante o ciclismo. Cinergis, Santa Cruz do Sul, v. 13, n. 1, p. 40-50, jan./abr. 2012. doi: 10.17058/cinergis.v13i1.2516.

CANDOTTI, C. T. et al. EMG signal behavior in human vastus lateralis, tibialis anterior and soleus muscles during fatigue. Brazilian Journal of Biomechanics, São Paulo, v. 5, n. 9, p. 15-19, Nov. 2004.

CARVALHO, W. M. Indoor cycling: guia prático para pedalar em casa. Belo Horizonte: Leitura, 2005.
CRUZ, C. F. Estudo eletromiográfico dos músculos bíceps femoral (porção longa), semitendinoso, semimembranoso e gastrocnêmio no ciclismo: comparação entre pedal de encaixe e pedal de presilha. $1998.68 \mathrm{f}$. Monografia (Graduação) - Faculdade de Educação Física da Universidade Estadual de Campinas, Campinas, 1998.

DIAS, M. R.; LIMA, J. R. P.; NOVAES, S. J. Cadência de pedalada no ciclismo: uma revisão de literatura. Revista Motricidade, Vila Real, v. 3, n. 1, p. 270-278, jan. 2007.

DIEFENTHAELER, F. et al. Ativação muscular durante a pedalada em diferentes posições do selim. Revista Brasileira de Cineantropometria e Desempenho Humano, Florianópolis, v. 10, n. 2, p. 161-169, abr./jun. 2008. doi: 10.5007/1980-0037.2008v10n2p161.

DIEFENTHAELER, F; VAZ, M. A. Aspectos relacionados à fadiga durante o ciclismo: uma abordagem biomecânica. Revista Brasileira de Medicina do Esporte, Niterói, v. 14, n. 5, p. 472-477, set./out. 2008. doi: 10.1590/ S1517-86922008000500014.

DOREL, S.; COUTURIER, A.; HUG, F. Influence of different racing positions on mechanical and electromyographic patterns during pedaling. Scandinavian Journal of Medicine and Science in Sports, Copenhagen, v. 19, n. 1, p. 44-54, Feb. 2009. doi: 10.1111/j.16000838.2007.00765.x.

FERMINO, F. R. Ciclismo de velocidade: uma proposta de controle da preparação física especial. 2008. 169 f. Dissertação (Mestrado) - Programa de Pós Graduação em Educação Física da Universidade Estadual de Campinas, Campinas, 2008.

FERREIRA, A. S; GUIMARÃES, F. S.; SILVA, J. G. Aspectos Metodológicos da eletromiografia da superfície: considerações sobre os sinais e processamentos para estudo da função neuromuscular. Revista Brasileira de Ciências do Esporte, Campinas, v. 31, n. 2, p. 11-30, jan. 2010.

KLEINPAUL, F. J. et al. Aspectos determinantes do posicionamento corporal no ciclismo: uma revisão sistemática. Revista Motriz, Rio Claro, v. 16, n. 4, p. 1013-1023, out./dez. 2010. doi: 10.5016/1980-6574.2010v16n4p1013. 
MESQUITA FILHO, R. B.; CASTRO, F. A. S. Análise eletromiográfica dos músculos dos membros inferiores no movimento da pedalada: uma revisão bibliográfica. EFDeportes Revista Digital, Buenos Aires, ano 16, n. 163, dez. 2011.

OKANO, A. H. et al. Respostas eletromiográficas dos músculos vasto lateral e reto femoral durante esforço intermitente anaeróbio em ciclistas. Revista Motriz, Rio Claro, v. 11, n. 1, p. 11-24, jan./abr. 2005.

PINZON, C. et al. Proposta metodológica para determinação da posição do selim aplicado ao ciclismo indoor: estudo de caso. p. 1-5, 2010. Disponível em: $<$ http://docplayer.com.br/7026195-Proposta-metodologica-para-determinacao-da-posicao-do-selim-aplicada-ao-ciclismo-indoor-estudo-de-caso.html>. Acesso em: 23 nov. 2016.
RIENDA, A.S. et al. Prevalência de lesões em praticantes de ciclismo indoor. EFDeportes Revista Digital, Buenos Aires, ano 17, n. 170, jul. 2012.

SURFACE ELECROMYOGRAPHY FOR THE NON-INVASIVE ASSESSMENT OF MUSCLES. Welcome at SE-

NIAM.org. Disponível em: <seniam.org>. Acesso em: 15 out. 2012.

SILVA, R. A. S.; OLIVEIRA, H. B. Prevenção de lesões no ciclismo indoor: uma proposta metodológica. Revista Brasileira Ciência e Movimento, Brasília, v. 10, n. 4, p. 7-18, out./dez. 2002.

SILVA, T.S. et al. Análise do nível de força dos músculos flexores e extensores do joelho de praticantes de ciclismo indoor. EFEDeportes Revista Digital, Buenos Aires, ano 17, n. 167, abr. 2012. 